\title{
Meta-analysis of Perceived Stress and its Association with Incident Coronary Heart Disease
}

\author{
Safiya Richardson, MD $^{\mathrm{a}}$, Jonathan A. Shaffer, $\mathrm{PhD}^{\mathrm{b}}$, Louise Falzon, PGDiplnf ${ }^{\mathrm{b}}$, David \\ Krupka, MA $^{\mathrm{b}}$, Karina W. Davidson, PhD $^{\mathrm{b}}$, and Donald Edmondson, $\mathrm{PhD}^{\mathrm{b}}$ \\ ${ }^{a}$ College of Physicians and Surgeons, Columbia University Medical Center, New York, NY, USA \\ ${ }^{\mathrm{b} C e n t e r}$ for Behavioral Cardiovascular Health, Columbia University Medical Center, New York, \\ NY, USA
}

\section{Abstract}

Most studies examining potential associations between psychological factors and cardiovascular outcomes have focused on either depression or anxiety. The effect of perceived stress on incident coronary heart disease (CHD) has yet to be reviewed systematically. We conducted a systematic review and meta-analysis of the association between perceived stress and incident CHD. Ovid MEDLINE and PsycINFO were searched as data sources. Prospective observational cohort studies were selected that measured self-reported perceived stress and assessed incident CHD at minimum of 6 months later. We extracted study characteristics and estimates of the risk of incident CHD associated with high perceived stress vs. low perceived stress. We identified 23 potentially relevant articles, of which 6 met our criteria $(\mathrm{N}=118,696)$. Included studies measured perceived stress with both validated measures and non-validated simple self-report surveys. Incident CHD was defined as new diagnosis of, hospitalization for, or mortality secondary to CHD. Metaanalysis yielded an aggregate risk ratio of 1.27 (95\% confidence interval $1.12-1.45$ ) for the magnitude of the relation between high perceived stress and incident CHD. In conclusion this meta-analysis suggests that high perceived stress is associated with a moderately increased risk of incident CHD.

\section{Keywords}

stress; perceived stress; coronary heart disease; ischemic heart disease

\begin{abstract}
Measures of stress in recent studies have been heterogeneous and included disparate definitions such as caring for a demented partner or spouse[1], reporting of lack of general psychological well-being[2], or reporting the occurrence of negative life events like divorce[3]. Types of chronic stress that have consistently been associated with increased cardiovascular risk are work-related stress[4] and marital stress[5]. However, it may be that perceived stress, the general perception that environmental demands exceed perceived capacity, regardless of source of the environmental demand, is also consistently associated
\end{abstract}

(C) 2012 Excerpta Medica, Inc. All rights reserved.

Corresponding author: Donald Edmondson, PhD, Telephone: 212-342-3674; Fax: 212-305-3172; dee2109@ columbia.edu, Mailing address: Center for Behavioral Cardiovascular Health, Columbia University Medical Center, 622 W 168 St, PH9-317, New York, NY 10032.

Publisher's Disclaimer: This is a PDF file of an unedited manuscript that has been accepted for publication. As a service to our customers we are providing this early version of the manuscript. The manuscript will undergo copyediting, typesetting, and review of the resulting proof before it is published in its final citable form. Please note that during the production process errors may be discovered which could affect the content, and all legal disclaimers that apply to the journal pertain. 
with incident coronary heart disease (CHD). To address this question, we conducted a systematic review of all prospective observational cohort studies that have asked participants to report perceived stress, and then followed those participants to assess incident CHD. Meta-analysis was used to derive an aggregate estimate of the risk ratio for the association between high perceived stress and incident CHD. Finally, we conducted sensitivity analyses to determine whether effect size estimates differed by study characteristics.

\section{Methods}

We sought to identify all studies that reported a valid estimate of the association between perceived stress and incident CHD diagnosis, hospitalization for CHD or mortality secondary to CHD. To be included, studies must have been prospective observational cohorts that featured a self-report assessment of perceived stress, on which participants could report frequency and/or intensity of stress. Only studies that used measures specifically referencing "stress," and not symptoms of psychological disorders such as depression, anxiety, or PTSD, were included in this review.

We searched the electronic databases Ovid MEDLINE and PsycINFO for potentially relevant articles. Dates included in the search were 1948 to July 21, 2011, the date the search was conducted. All relevant subject heading and free text terms were used to represent stress and CHD, and the sets of terms were combined with AND. Terms for MEDLINE to represent stress included: exp Stress, Psychological/OR ((Psycho\$ or mental\$ or emotion\$ or financ\$ or marital or relationship\$ or social $\$$ or job\$ or occupation $\$$ or employ $\$$ or work\$ or global) adj5 (stress\$ or distress\$)).tw. OR exp Stress Disorders, Traumatic/OR ptsd.tw. OR (post-traumatic OR (post adj traumatic)).tw OR posttraumatic.tw. Terms to represent CHD included: exp Myocardial Ischemia/OR ((coronary or heart or isch?emi\$ or myocardial or cardiac) adj3 (disease\$ or syndrome $\$$ or attack\$ or event\$)).tw. OR (acs or acd or chd or cad).tw. OR ((post adj acs) or postasc).tw. These terms were adapted for PsycINFO.

Additional records were identified by scanning the references lists of relevant studies and reviews and by employing the Related Articles feature in PubMed and the Cited Reference Search in ISI Web of Science.

To determine the studies to be assessed further, 2 authors (SR, LF) independently read the abstract and/or titles of every record retrieved for the selection criteria listed below. All potentially relevant articles were investigated as full text. Differences in opinion were resolved by consensus.

We abstracted effect size estimates and study characteristics of those studies describing the association of perceived stress to incident CHD diagnosis, hospitalization for CHD, and mortality secondary to CHD (Table 1). For multiple publications from the same cohort, we chose the publication with the largest sample size and the most appropriate outcome measure (e.g., Nielson et al. (2006) was included because it measured incident CHD diagnosis, whereas Nielsen et al. (2008) measured death secondary to CHD without excluding participants with a previous diagnosis of heart disease). All studies were coded for the following characteristics: sample size, years of follow-up, specific measure of perceived stress and scoring procedure, cardiovascular outcomes measured, sample age, gender, and race/ethnicity, year of publication, number of covariates and adjustment for psychological variables.

Comprehensive MetaAnalysis (version 2, BioStat Software, Engelwood, NJ) served as the statistical platform for completing all statistical tests and producing associated graphic results. We calculated an aggregate effect size in the form of a risk ratio associated with high 
vs. low perceived stress on incident CHD diagnosis, hospitalization for CHD, or mortality secondary to CHD.

Log-transformed risk ratios and 95\% confidence intervals (CIs) were calculated for each study using the reported effect size and estimates of the standard error (SE) of the effect size from data reported in the article. To assess heterogeneity in effect size estimates across studies, we calculated Cochran's Q, which is the weighted sum of squared differences between individual study effects and the pooled effect across studies, even though it has low power for detecting heterogeneity when the number of studies in a meta-analysis is small.[6] We also report $\mathrm{I}^{2}$, which is the percent of between-study variance in effect sizes that is due to heterogeneity rather than chance.[7] When articles reported multiple models, we selected the effect size from the most fully adjusted model. To address possible publication bias, we examined the funnel plot associated with the study effect sizes and calculated a fail-safe $\mathrm{N}$. [8]

In addition to the overall fixed- and random-effects meta-analysis models, we used sensitivity analyses to assess possible moderator effects for the association of perceived stress to CHD outcomes across a number of methodological factors. These factors included the measure used to assess perceived stress and the type of CHD or mortality outcome. Further, meta-regression analysis was used to test the association between effect size and study publication date and mean sample age. Separate estimates for men and women were available in 3 studies, so we were able to compare the association by gender. Similarly, separate estimates were available for cardiac events with and without angina in 3 studies, so we also compared those effect size estimates.

\section{Results}

Among 23 articles identified in our initial search, 19 required full reading after duplicate records were excluded. Of these 19 , six met our criteria for inclusion ( 5 articles from the initial search[9-13] and another 1 article identified from references[14]) (Figure 1).

All included studies completed recruitment and assessment of baseline characteristics between 1970 and 1990. Follow-up periods ranged from 36 to 255 months with an average of 165.9 months of follow-up (or 13.8 years). Studies were conducted in Sweden (2), Scotland (1), Australia (1), Denmark (1) and Japan (1). Two studies enrolled men only and 1 enrolled females only. For the remaining 3 studies, the average percent of the sample that were men was $56.4 \%$. Mean age for all study participants was $55.0 \pm 10.18$ years. Two studies used validated measures of perceived stress, and 4 asked 1 or 2 questions about the experience of daily stress (Table 2).

One of the included studies used the Perceived Stress Scale, which was designed to measure the degree to which individuals appraise situations in their lives as stressful.[15] The Perceived Stress Scale uses paper and pencil self-report, takes about 5 minutes to complete and evaluates the degree to which people find life unpredictable, uncontrollable, or overloaded. The validity and reliability were assessed in a large national probability sample $(\mathrm{N}=2,387)$ in the United States.[16] Another study used the Reeder Stress Inventory, which consists of 4 statements about feelings of strain, tension, exhaustion and stress in association with daily activities. Respondents rate the extent to which each of these 4 statements is true using a 4 point Likert format. This measure was validated in a cross-sectional investigation $(\mathrm{N}=1,717)$ in the United Kingdom.[17]

The remaining studies asked 1 or 2 questions about intensity and/or frequency of daily stress. The nature of these questions was similar to questions included in the validated measures of perceived stress. The thresholds for labeling participants as experiencing high 
stress were similar across all studies where participants essentially had to directly report either intense or frequent feelings of stress (Table 2).

Cardiac outcomes included death from CHD, incident CHD diagnoses and events (including angina) and hospital admission for CHD. Every study controlled for traditional cardiac risk factors (e.g., age, blood pressure, smoking, cholesterol), 3 studies additionally controlled for socioeconomic status, and another study controlled for depression and anxiety symptoms.

The aggregated effect size associated with high vs. low perceived stress for incident CHD was a risk ratio of 1.27 (95\% [CI], 1.12-1.45) in the random effects model (Figure 2). There was no statistically significant between-study heterogeneity in effect size estimates, $Q(5)=$ 9.54, $\mathrm{p}=.09 .[18,19]$ However, because the P-value for the significance test of the assessment of heterogeneity was $<.10$, we decided to report the random effects estimate and conduct our a priori sensitivity analyses by study characteristics, with the understanding that the lack of heterogeneity and small number of studies limited our power to detect associations between study characteristics and effect size estimates.

Although publication bias is difficult to assess with 6 studies, the funnel plot suggested that studies in which there was no relationship between high perceived stress and clinical outcomes may have been missing (Figure 3). However, the fail-safe $\mathrm{N}$ suggested that 40 additional studies with no relationship between perceived stress and clinical outcomes would have needed to exist for the risk ratio for the true association to be 1 . This fail-safe $\mathrm{N}$ indicates that our meta-analytic finding is robust to publication bias, as a generally accepted rule of thumb for interpreting fail-safe $\mathrm{N}$ is that a meta-analytic result is robust to publication bias if $\mathrm{N} \geq 5 k+10$, where $k$ is the number of studies included in the metaanalysis, and so 40 in this case. [8]

There was no difference in effect size estimates by type of stress measure, either validated or non-validated, $\mathrm{p}=0.98$.

One study used death as the primary $\mathrm{CHD}$ outcome $(\mathrm{N}=73,414$; risk ratio $=1.51 ; 95 \% \mathrm{CI}=$ $0.97-2.36)$. The effect size from that study did not differ $(Q(1)=0.59, p=0.44)$ from those of studies with fatal and non-fatal incident CHD events included as the primary outcome $(\mathrm{N}=45,282$; risk ratio $=1.26 ; 95 \% \mathrm{CI}=1.10-1.44)$.

Three studies provided separate effect size estimates for CHD events including angina vs myocardial infarction (MI) only. The comparison of effect size estimates for the overall CHD vs MI only in those 3 studies showed that they were not significantly different from one another, $Q(1)=2.85, p=.09$. However, while the aggregate point estimate for the combined CHD outcome across the 3 studies was 1.17, $\mathrm{p}=0.02$ (95\% CI, 1.06-1.29), the aggregated MI only estimate was 1.004 (95\% CI, 0.87-1.16), $\mathrm{p}=0.95$.

Three studies calculated estimates separately for men and women. We compared estimates for men vs. women across those 3 studies and found no significant difference between approximated risk for the sexes $(\operatorname{men}=1.19$, women $=1.30 ; \mathrm{Q}($ between $)=0.30, \mathrm{p}=0.59$ ).

The mean age of the participants varied from 44 to 72.5 years. Older mean sample age was significantly related to a greater effect size across the studies, and explained $46 \%$ of the variance in effect sizes, $Q($ model $)=4.38, p=0.03 ; Q($ total $)=9.54, p=.09$.

Meta-regression analysis showed that date of publication was unrelated to effect size estimates, $Q($ model $)=2.51, p=0.11$. 
In an additional meta-regression analysis, amount of covariate adjustment, which ranged from 9-17 covariates, was unrelated to effect size estimates, $Q$ (model) $=0.48, p=0.49$. Two studies adjusted for at least 1 psychological covariate (Iso et al. (2002): anger, hopelessness, senses of joyfulness, being trusted, fulfillment; Rosengren et al. (1991): registration for alcohol abuse). Inclusion of psychological covariates compared to noninclusion of psychological covariates did not significantly affect the effect size estimates, $Q$ $(1)=3.10, p=0.08$, but the mean effect size of the 2 studies that included such covariates was $1.50(95 \% \mathrm{CI}=1.23-1.84)$, whereas the mean effect size for the remainder of studies was $1.21(95 \% \mathrm{CI}=1.06-1.38)$.

\section{Discussion}

This is the first meta-analytic review of the association of perceived stress to incident CHD. By combining results from 6 large prospective observational cohort studies representing 118,696 participants, followed for an average of more than a decade, we found that high perceived stress is associated with a risk ratio of 1.27 for incident CHD. It is useful to compare the magnitude of the increased risk associated with high perceived stress to those of traditional cardiovascular risk factors. For hyperlipidemia, there is a $21 \%$ increase in cardiovascular events with every $39 \mathrm{mg} / \mathrm{dL}$ increase in LDL cholesterol.[20] For hypertension, risk for cardiovascular disease increases by $200 \%$ with every additional increment of 20/10 $\mathrm{mmHg}$ above a blood pressure of 115/75 mmHg.[21] For smoking, the risk of acute MI increases by 5.6\% with every additional cigarette smoked per day.[22] Based on these approximations of risk, high perceived stress, which was associated with a $27 \%$ increase in CHD risk in this meta-analysis, could be thought of as the equivalent of a $50 \mathrm{mg} / \mathrm{dL}$ increase in LDL, a $2.7 / 1.4 \mathrm{mmHg}$ increase in blood pressure or 5 more cigarettes per day.

The mechanism linking perceived stress to adverse cardiovascular outcomes is likely multifactorial. Possibilities include increased activity of the hypothalamic pituitary axis[23], increased sympathetic outflow[24] or altered behaviors causing insulin resistance and central obesity[25]. Notably, many of the studies included in this review controlled for indirect measures at baseline of increased activity of the hypothalamic pituitary axis, increased sympathetic outflow and altered behaviors. It is possible that these mechanisms increase across time, and so need to be measured with both more precision, and as timevarying covariates. The exact mechanism behind the association between stress and CHD is still unclear, but it is not required for recognizing the significance of the association.

Randomized controlled trials of stress interventions and cardiac outcomes have shown efficacy for treating "stress" even though definitions of stress have varied. A recent metaanalysis of 43 studies showed that psychological interventions targeted to stress reduction (including cognitive behavioral therapy, yoga and muscle relaxation techniques) decreased 2-year mortality in men and event recurrence in all CHD patients by 27\%.[26] No trials have yet examined the effects of stress reduction on CHD incidence.

The results of this meta-analysis have important limitations. Because few studies have examined perceived stress and cardiac outcomes, only 6 studies could be included. To address the issue of publication bias, we calculated a fail-safe $\mathrm{N}$ which suggested that a substantial number of unpublished studies would have to exist for there to be no true relationship. Also, we contacted authors of included studies about relevant unpublished data, but this did not generate any studies that fit our inclusion criteria. Further, although only 6 studies fit our inclusion criteria, these studies included prospective population-based studies with a very large aggregate sample size. 
Most of the included studies did not adjust for psychological variables like depression and anxiety, which are known to be associated with both perceived stress and CHD. Notably, the effect size for the 2 studies that did adjust for psychological variables did not differ from those that did not control for these constructs. However, there is clearly a need for studies that can better control for these important variables.

Lastly, as most of the studies were conducted in racially and ethnically homogenous European and Asian countries, there was very little inclusion of those from different geographic and ethnic backgrounds. Racial and ethnic minority populations may have unique experiences and sequelae of perceived stress and findings from these populations might differ from those reported here.

\section{Acknowledgments}

Grant Support: This work was supported by grants HL-088117 and CA-156709, and in part by Columbia University's CTSA grant No.UL1RR024156, from the National Institutes of Health, Bethesda, Maryland, United States. Its contents are solely the responsibility of the authors and do not necessarily represent the official view of the National Center for Research Resources or the National Institutes of Health.

\section{References}

1. Vitaliano PP, Scanlan JM, Zhang J, Savage MV, Hirsch IB, Siegler IC. A path model of chronic stress, the metabolic syndrome, and coronary heart disease. Psychosom Med. 2002; 64:418-435 [PubMed: 12021416]

2. Rasul F, Stansfeld S, Hart C, Smith GD. Psychological distress, physical illness, and risk of coronary heart disease. J Epidemiol Community Health. 2005; 59:140-145. [PubMed: 15650146]

3. Jenkinson C, Madeley R, Mitchell J, Turner I. The influence of psychosocial factors on survival after myocardial infarction. Public Health. 1993; 107:305-317. [PubMed: 8248465]

4. Eller NH, Netterstrøm B, Gyntelberg F, Kristensen TS, Nielsen F, Steptoe A, Theorell T. Workrelated psychosocial factors and the development of ischemic heart disease: a systematic review. Cardiol Rev. 2009; 17:83. [PubMed: 19367150]

5. Eaker ED, Sullivan LM, Kelly-Hayes M, D’Agostino RB, Benjamin EJ. Marital status, marital strain, and risk of coronary heart disease or total mortality: The Framingham offspring study. Psychosom Med. 2007; 69:509-513. [PubMed: 17634565]

6. Gavaghan DJ, Moore RA, McQuay HJ. An evaluation of homogeneity tests in meta-analyses in pain using simulations of individual patient data. Pain. 2000; 85:415-424. [PubMed: 10781914]

7. Higgins J, Thompson SG, Deeks JJ, Altman DG. Measuring inconsistency in meta-analyses. BMJ. 2003; 327:557. [PubMed: 12958120]

8. Orwin RG. A Fail-Safe N for Effect Size in Meta-Analysis. J Educ Stat. 1983; 8:157-159.

9. Iso H, Date C, Yamamoto A, Toyoshima H, Tanabe N, Kikuchi S, Kondo T, Watanabe Y, Wada Y, Ishibashi T, Suzuki H, Koizumi A, Inaba Y, Tamakoshi A, Ohno Y. Perceived mental stress and mortality from cardiovascular disease among Japanese men and women: the Japan Collaborative Cohort Study for Evaluation of Cancer Risk Sponsored by Monbusho (JACC Study). Circulation. 2002; 106:1229-1236. [PubMed: 12208798]

10. MacLeod J, Davey Smith G, Heslop P, Metcalfe C, Carroll D, Hart C. Limitations of adjustment for reporting tendency in observational studies of stress and self reported coronary heart disease. $\mathrm{J}$ Epidemiol Community Health. 2002; 56:76-77. [PubMed: 11801624]

11. Nielsen NR, Kristensen TS, Prescott E, Larsen KS, Schnohr P, Gronbaek M. Perceived stress and risk of ischemic heart disease: causation or bias? Epidemiology. 2006; 17:391-397. [PubMed: 16755264]

12. Rosengren A, Tibblin G, Wilhelmsen L. Self-perceived psychological stress and incidence of coronary artery disease in middle-aged men. Am J Cardiol. 1991; 68:1171-1175. [PubMed: 1951076]

13. Strodl E, Kenardy J, Aroney C. Perceived stress as a predictor of the self-reported new diagnosis of symptomatic CHD in older women. Int J Behav Med. 2003; 10:205-220. [PubMed: 14525717] 
14. Öhlin B, Nilsson P, Nilsson JÅ, Berglund G. Chronic psychosocial stress predicts long-term cardiovascular morbidity and mortality in middle-aged men. Eur Heart J. 2004; 25:867-873. [PubMed: 15140535]

15. Cohen S, Kamarck T, Mermelstein R. A global measure of perceived stress. J Health Soc Behav. 1983:385-396. [PubMed: 6668417]

16. Cohen, S.; Williamson, G. Perceived stress in a probability sample of the United States. In: Spacapan, S.; Oskamp, S., editors. The social psychology of health: Claremont Symposium on Applied Social Psychology. Newbury Park, CA: Sage; 1988.

17. Metcalfe C, Smith GD, Wadsworth E, Sterne JAC, Heslop P, Macleod J, Smith A. A contemporary validation of the Reeder Stress Inventory. Br J Health Psychol. 2003; 8:83-94. [PubMed: 12643818]

18. Fleiss JL, Gross AJ. Meta-analysis in epidemiology, with special reference to studies of the association between exposure to environmental tobacco smoke and lung cancer: a critique. J Clin Epidemiol. 1991; 44:127-139. [PubMed: 1995774]

19. Ades A, Lu G, Higgins J. The interpretation of random-effects meta-analysis in decision models. Med Decis Making. 2005; 25:646-654. [PubMed: 16282215]

20. Yusuf S, Lonn E, Bosch J. Lipid lowering for primary prevention. Lancet. 2009; 373:1152-1155. [PubMed: 19345816]

21. Chobanian AV, Bakris GL, Black HR, Cushman WC, Green LA, Izzo JL, Jones DW, Materson BJ, Oparil S, Wright JT. Seventh report of the joint national committee on prevention, detection, evaluation, and treatment of high blood pressure. Hypertension. 2003; 42:1206-1252. [PubMed: 14656957]

22. Teo KK, Ounpuu S, Hawken S, Pandey M, Valentin V, Hunt D, Diaz R, Rashed W, Freeman R, Jiang L. Tobacco use and risk of myocardial infarction in 52 countries in the INTERHEART study: a case-control study. Lancet. 2006; 368:647-658. [PubMed: 16920470]

23. Tsigos C, Chrousos GP. Hypothalamic-pituitary-adrenal axis, neuroendocrine factors and stress. J Psychosom Res. 2002; 53:865-871. [PubMed: 12377295]

24. Fredrikson M, Matthews KA. Cardiovascular responses to behavioral stress and hypertension: A meta-analytic review. Ann Behav Med. 1990; 12:30-39.

25. Räikkönen K, Keltikangas-Järvinen L, Adlercreutz H, Hautanen A. Psychosocial stress and the insulin resistance syndrome. Metabolism. 1996; 45:1533-1538. [PubMed: 8969288]

26. Linden W, Phillips MJ, Leclerc J. Psychological treatment of cardiac patients: a meta-analysis. Eur Heart J. 2007; 28:2972-2984. [PubMed: 17984133] 


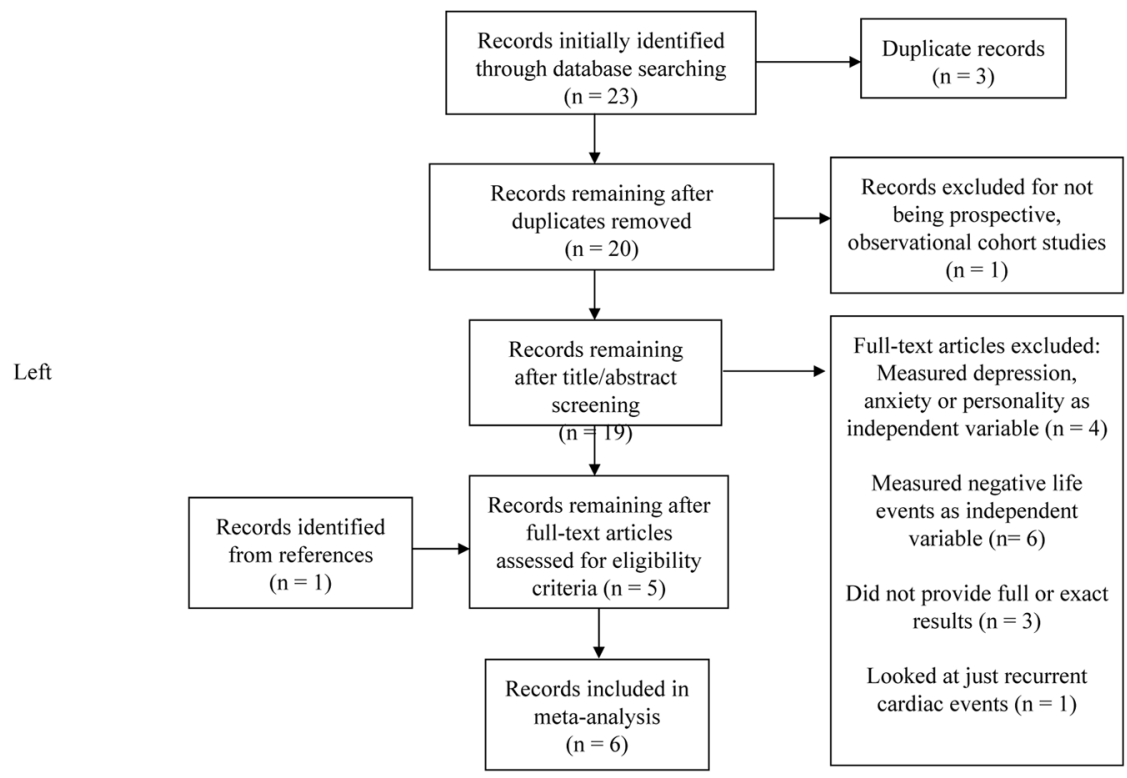

Bottom

Figure 1.

Search strategy flowchart 


\begin{tabular}{clccc} 
& Study name & \multicolumn{3}{c}{ Statistics for each study } \\
& & $\begin{array}{c}\text { Risk } \\
\text { ratio }\end{array}$ & $\begin{array}{c}\text { Lower } \\
\text { limit }\end{array}$ & $\begin{array}{c}\text { Upper } \\
\text { limit }\end{array}$ \\
& Left & 1.51 & 0.97 & 2.36 \\
& Iso, 2002 & 1.00 & 0.76 & 1.32 \\
& Macleod, 2002 & & \\
& Nielsen, 2006 & 1.24 & 1.07 & 1.44 \\
& Ohlin, 2004 & 1.16 & 1.02 & 1.32 \\
& Rosengren, 1991 & 1.50 & 1.19 & 1.89 \\
& Strodl, 2003 & 1.67 & 1.17 & 2.38 \\
& & 1.27 & 1.12 & 1.45
\end{tabular}

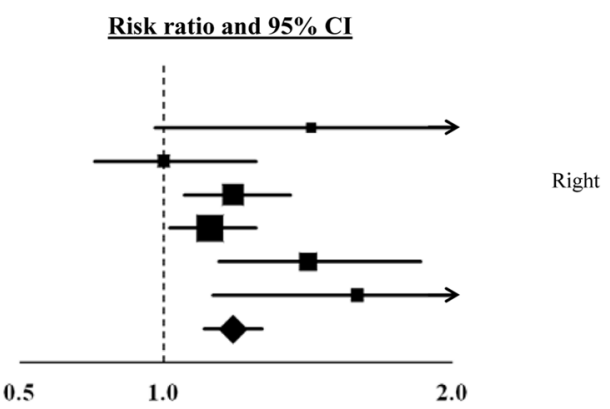

Bottom

Figure 2.

High perceived stress and coronary heart disease incidence estimates

Note: The area of each square is proportional to the study's weight in the meta-analysis, and each line represents the confidence interval around the estimate. The diamond represents the aggregate estimate, and its lateral points indicate confidence intervals for this estimate. 


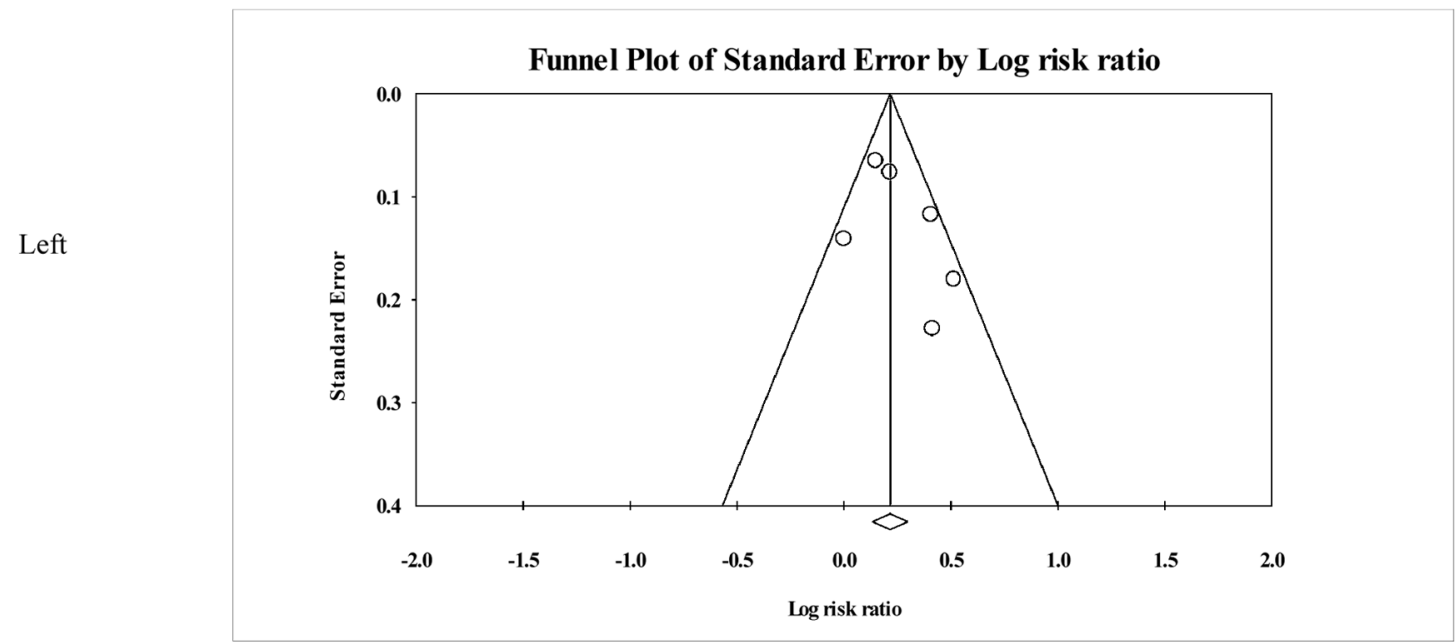

Bottom

Figure 3.

Funnel plot to assess publication bias across incidence studies 


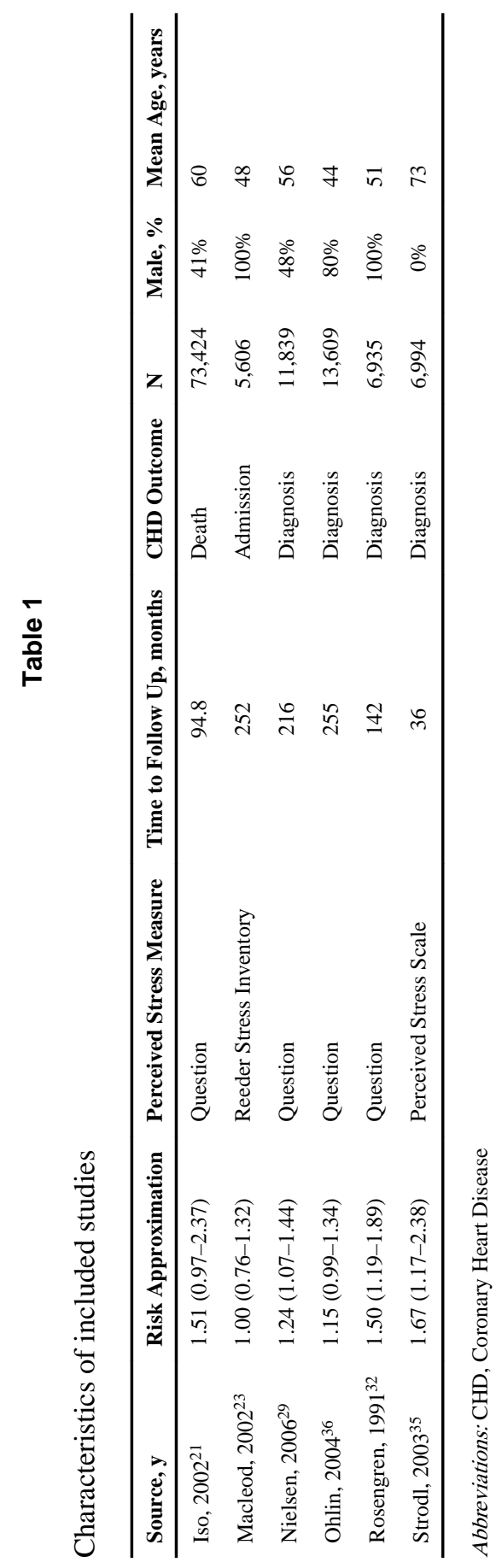




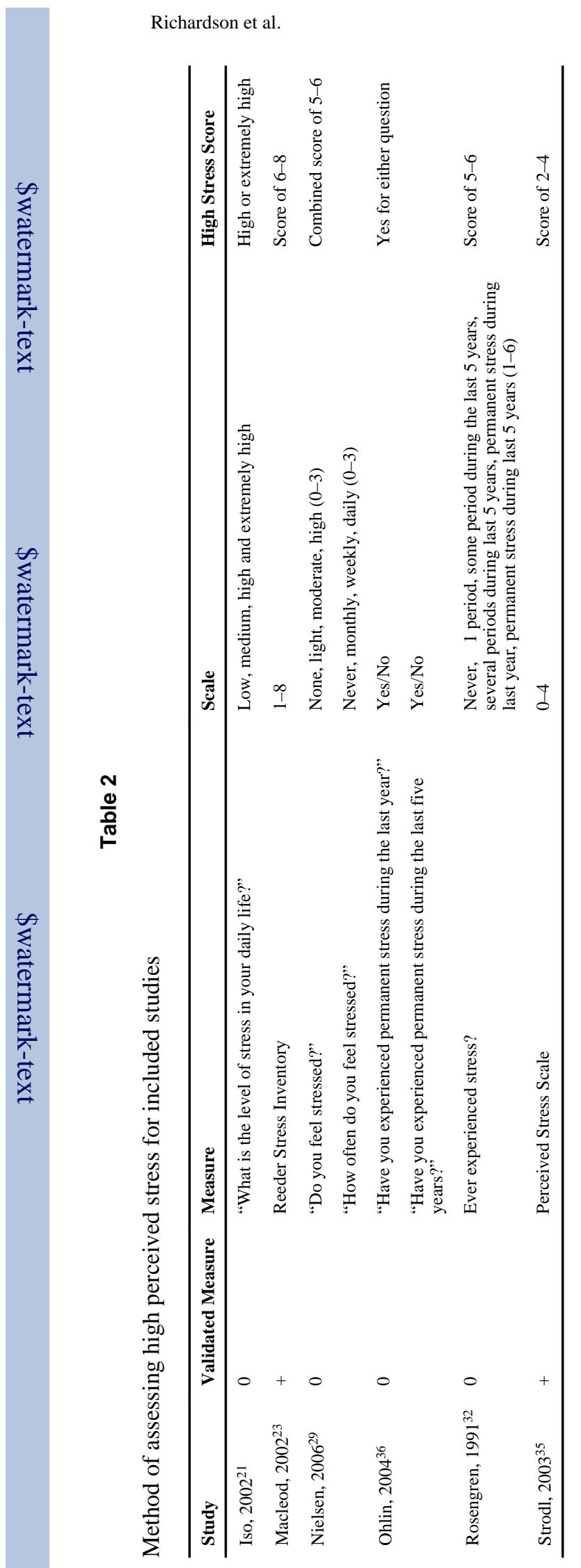

Page 12 